\title{
THE COINCIDENCE PROBLEM FOR COMPOSITIONS OF SET-VALUED MAPS
}

\author{
H. BEN-EL-MechaIEKH
}

The main purpose of this work is to give a general and elementary treatment of the fixed point and the coincidence problems for compositions of set-valued maps with not necessarily locally convex domains and to display, once more, the central rôle played by the selection property.

\section{INTRODUCTION}

The following coincidence theorem was proved by Granas and Liu in [17]:

THEOREM 0 . Let $X, Y$ be convex subsets of topological vector spaces, and $A, B: X \rightarrow Y$ two set-valued maps satisfying:

(i) $A$ is upper semicontinuous (usc) and has non-empty compact acyclic values;

(ii) $B$ has non-empty convex values and open fibres.

If $A$ is compact, then $A$ and $B$ have a coincidence, that is, there exists $\left(x_{0}, y_{0}\right) \in X \times Y$ with $y_{0} \in A\left(x_{0}\right) \cap B\left(x_{0}\right)$.

[Recall that a nonempty compact space is acyclic if all its reduced Cech homology groups over $Q$ vanish.]

The proof of Theorem 0 relies on a Lefschetz-type fixed point theorem of Gòrniewicz and Granas [14] which is itself based on sophisticated homological machinery. One of our concerns was to provide a simple proof of the convex case, that is when $A$ is usc and has non-empty convex compact values (such a map will be called a $K$-map). In doing so, we were driven to give an elementary proof of a fairly general fixed point theorem for compositions of $\mathbf{K}$-maps defined on a general extension space containing the locally convex and the not necessarily locally convex cases. Weaker results were recently and independently obtained by Lassonde [24]. However, our fixed point theorems are different from Lassonde's in the sense that the spaces are more general and an approximate selection technique is used here rather then the approximation by simplicial maps (see Kakutani [21], $\mathrm{Ha}$ [18], Lassonde [24]). We are thus able to deduce some general coincidence theorems for compositions of set-valued maps.

Received 28 June 1989

This research was supported in part by a grant from the National Council of Canada.

Copyright Clearance Centre, Inc. Serial-fee code: 0004-9729/90 \$A2.00+0.00. 
Parts of this paper are taken from the author's thesis [3] and are motivated by a joint work with Deguire and Granas [6] to whom the author expresses his appreciation.

\section{DEFinitions and PRELIMINARY RESUlts}

Let $X$ and $Y$ be two sets. A set-valued map $A: X \rightarrow Y$ is a map that associates with any $x \in X$ a subset $A(x)$ of $Y$. The subsets $A(x)$ of $Y$ are called the values of $A$ and the subsets $A^{-1}(y)=\{x \in X \mid y \in A(x)\}$ of $X$ are the fibres of $A$; the map $A^{-1}: Y \rightarrow X$ is the inverse of $A$.

In what follows, set-valued maps, simply called maps, are denoted by capital letters while single valued functions, called functions, are denoted by small letters. The graph of map $A: X \rightarrow Y$ is the set $\Gamma_{A}=\{(x, y) \in X \times Y \mid y \in A(x)\}$. Given two $A, B: X \rightarrow Y$, $B$ is said to be a multiselection or simply selection of $A$ if $\Gamma_{B} \subseteq \Gamma_{A}$ that is $B(x) \subseteq A(x)$ for each $x \in X$; similarly, a function $s: X \rightarrow Y$ is a selection of $A$ if $\Gamma, \subseteq \Gamma_{A}$.

We say that $A$ and $B$ have a coincidence if $\Gamma_{B} \cap \Gamma_{A} \neq \emptyset$. If $A$ is a self map, $x \in X$ is a fixed point for $A$ if $x \in A(x)$; we denote by $\operatorname{Fix}(A)$ the set of all fixed points of $A$. For a subset $K$ of a topological space $X$, we denote by $\operatorname{Cov}_{X}(K)$ the directed set of all coverings of $K$ by open sets of $X\left(\operatorname{Cov}(X)=\operatorname{Cov}_{X}(X)\right)$. Given a map $A: X \rightarrow X$ and $\alpha \in \operatorname{Cov}(X)$, a point $x \in X$ is said to be an $\alpha$-fixed point of $A$ if there exists a member $U \in \alpha$ such that (i) $x \in U$ and (ii) $A(x) \cap U \neq \emptyset$. Given two maps $A, B: X \rightarrow Y$ and $\alpha \in \operatorname{Cov}(Y), A$ and $B$ are said to be $\alpha$-close, if for any $x \in X$, there exist $U_{x} \in \alpha, y \in A(x) \cap U_{x}$ and $y^{\prime} \in B(x) \cap U_{x}$.

Given a class $\mathbf{A}$ of maps, we define

$$
\begin{gathered}
\mathbf{A}(X, Y)=\{A \in \mathbf{A} \mid A: X \rightarrow Y\} ; \mathbf{A}(X)=A(X, X) ; \mathbf{A}^{-}=\left\{A \mid A^{-1} \in \mathbf{A}\right\} \\
\mathbf{A}_{c}=\left\{A=A_{m} A_{m-1} \ldots A_{1} \mid A_{i} \in \mathbf{A}\right\}, \mathcal{F}_{\mathbf{A}}=\{X \mid(\forall A \in \mathbf{A}(X))(F i x(A) \neq \emptyset)\} . \\
\mathbf{A}^{-} \text {is called the inverse class of } A ; \text { clearly }\left(\mathbf{A}^{-}\right)^{-}=\mathbf{A} \\
\text { and } \operatorname{Fix}(A) \neq \emptyset \Leftrightarrow \operatorname{Fix}\left(A^{-1}\right) \neq \emptyset .
\end{gathered}
$$

Let $\mathcal{C}$ denote the class of all continuous functions. We say that a class of maps $\mathbf{A}$ is regular if (i) $A \times B \in \mathbf{A}$ for all $A, B \in \mathbf{A}$ and (ii) the composition $A t \in \mathbf{A}$ for all $A \in \mathbf{A}$ and $t \in \mathcal{C}$.

The following is immediate:

LEmмA 1.1. Given a regular class $\mathbf{A}$ and $X, Y$ two sets such that $X \times Y \in \mathcal{F}_{\mathrm{A}}$. Then

(i) $\Gamma_{B} \cap \Gamma_{A} \neq \emptyset$ whenever $A \in \mathbf{A}(X, Y)$ and $B \in \mathbf{A}-(X, Y)$ and

(ii) $\operatorname{Fix}(B A) \neq 0$ whenever $A \in \mathbf{A}(X, Y)$ and $B \in \mathbf{A}(Y, X)$. 
In view of this result, if all the spaces are "well behaved" (for example locally convex) then the existence of fixed point for compositions, and coincidence between inverse classes, are trivial. But if the intermediate spaces are not "nice" (for example not locally convex), then the previous lemma does not apply. The study of this case is the object of this paper.

We recall now some useful properties of maps (for definitions and basic properties of semicontinuity of maps, the reader is referred to Aubin and Cellina [2] and Berge $[7]$.

Lemma 1.2. Let $X$ be a regular topological space and $A: X \rightarrow X$ an upper semicontinuous map with closed values. Assume that there exists a cofinal family of coverings $\vartheta \subseteq \operatorname{Cov}(X)$ such that $A$ has an $\alpha$-fixed point for every $\alpha \in \vartheta$. Then $A$ has a fixed point.

Proof: Suppose that Fix $(A)=\emptyset$. For each $x \in X$, there exist open sets $U_{x} \in$ $\vartheta_{X}(x)$ (the family of all open neighbourhoods of $x$ in $X$ ) and $V_{x} \supseteq A(x)$ such that $V_{x} \cap U_{x}=\emptyset$ and $A\left(U_{x}\right) \supseteq V_{x}$. Putting $\beta=\left\{U_{x} \mid x \in X\right\}$ we get a covering of $X$ such that $A$ has no $\beta$-fixed point. If $\alpha$ is a member of $\vartheta$ that refines $\beta$, then $A$ has no $\alpha$-fixed point, which is a contradiction.

LEMMA 1.3. If the following diagram commutes

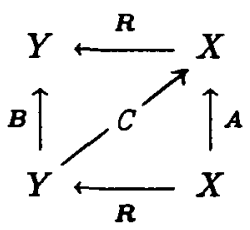

then $A$ has a fixed point if and only if $B$ has a fixed point.

For the sake of simplicity, we shall restrict our settings to the class of Hausdorff topological vector spaces (denoted subsequently by t.v.s.) even though the main results can be stated in the larger class of affine spaces in the sense of Dugundji [9] or convex spaces in the sense of Lassonde [23]. When the existence of separating linear functionals is needed, we shall merely require a locally convex structure rather than the sufficient and weaker condition of the existence of sufficiently many linear functionals.

\section{BASIC CLASSES OF MAPS}

We now define the classes of maps which will be studied in the sequel.

In what follows, $X$ and $Y$ are subsets of topological vector spaces $E$ and $F$, and, for any subset $K$ of $E$, conv $(K)$ (respectively $\overline{c o n v}(K)$ ) denotes the convex (respectively convex closed) envelope of $K$ in $E$. 
Define the classes:

$\mathcal{C}_{\Delta}(X, Y)=\left\{s=\in \mathcal{C}(X, Y) \mid\left(\exists\left\{y_{1}, y_{2}, \ldots, y_{n}\right\} \subseteq Y\right)\left(s(X) \subseteq \operatorname{conv}\left(y_{1}, y_{2}, \ldots, y_{n}\right)\right)\right\}$

$A \in \mathbf{M}(X, Y) \Leftrightarrow A / K$ has a selection $s \in \mathcal{C}_{\Delta}(K, Y)$ for every compact $K$ of $X$.

Clearly, $\mathcal{C}_{\Delta} \subseteq \mathbf{M}$

$A \in \mathbf{S}(X, Y) \Leftrightarrow A / K$ has a selection $s \in \mathcal{C}(K, Y)$ for every paracompact $K$ of $X$.

$A \in \mathrm{F}(X, Y) \Leftrightarrow$ (i) $A$ has non-empty convex values; (ii) $A$ has open fibres.

$A \in \Phi(X, Y) \Leftrightarrow\left\{\begin{array}{c}\text { (i) } A \text { has convex values; } \\ \text { (ii) } A \text { has a selection } \tilde{A} \text { with non-empty values and } \\ \text { open fibres. }\end{array}\right.$

Such a selection is called an admissible selection. Lassonde [23] remarked that every $\boldsymbol{\Phi}$-map has an $\mathbf{F}$-selection (indeed, for a given $A \in \Phi$, the map $\tilde{A}$ defined by $\widetilde{A}(x)=\bigcup_{V \in \vartheta(x)} \bigcap_{x \in V} A(z)$ is an F-selection of $\left.A\right)$.

$A \in \mathbf{K}(X, Y) \Leftrightarrow$ (i) $A$ is usc; (ii) $A$ has non-empty convex compact values.

$A \in \mathrm{V}(X, Y) \Leftrightarrow$ (i) $A$ is usc; (ii) $A$ has non-empty compact acyclic values.

$A \in \mathbf{L}(X, Y) \Leftrightarrow$ (i) $A$ is lsc; (ii) $A$ has non-empty closed convex values.

$$
A \in \mathbf{L}_{w}(X, Y) \Leftrightarrow\left\{\begin{array}{r}
\text { there exist two subsets } Z, C \subseteq X, \text { with } \operatorname{dim}_{X}(Z) \leqslant 0 \\
\text { and } C \text { countable such that: } \\
\text { (i) } A \text { is lsc; } \\
\text { (ii) } A(x) \text { is closed in } Y \text { for each } x \in X \backslash C ; \\
\text { (iii) } \overline{A(x)} \text { is convex for each } x \in X \backslash Z .
\end{array}\right.
$$

[Here $\operatorname{dim}_{X}(Z)$ denotes the topological dimension of $Z$ in $X$, see Hurewicz and Wall$\operatorname{man},[20]$.]

Of course, $\mathbf{F} \subset \mathbf{T}, \mathbf{K} \subset \mathbf{V}$, and $\mathbf{L} \subset \mathbf{L}_{\boldsymbol{w}}$. The class $\mathbf{F}$ was introduced and studied by Fan [11] and subsequently by Browder [8]. In special cases, the classes $K$ and $V$ were considered by Kakutani [21] and Vietoris [31] respectively. The classes S, L and $\mathbf{L}_{\boldsymbol{w}}$ were introduced and studied by Michael $[25,26]$.

LEMMA 2.1. (i) The classes $\mathbf{M}, \mathbf{F}, \mathbf{T}, \mathbf{K}, \mathbf{V}$ are regular,

(ii) $\mathbf{M}_{c}=\mathbf{M}$.

Thus, for a suitable choice of spaces, the existence of fixed points for compositions and of coincidences for these classes follows immediately from Lemma 1.1. 
REMARK. The class $\mathbf{S}$ is not regular. However, since the transpositon $t$ in the proof of Lemma 1.1 is a homeomorphism (and thus closed), and since paracompactness is invariant under closed surjections, $\mathbf{S}$ satisfies the conclusions of Lemma 1.1.

\section{Proposition 2.2. ([5]). $\Phi \subseteq M \cap S$.}

Proof: Let $A \in \Phi(X, Y), \tilde{A}$ be an admissible selection of $A$ and $K \subseteq X$ be compact. Since $\tilde{A}$ has non-empty values, $\alpha=\left\{\tilde{A}^{-1}(y) \mid y \in Y\right\} \in \operatorname{Cov} X(K)$. Now consider a partition of unity $\left\{\lambda_{i} \mid i=1,2, \ldots, n\right\}$ subordinated to a finite subcovering $\left\{\tilde{A}^{-1}\left(y_{i}\right) \mid i=1,2, \ldots, n\right\}$ of $\alpha$ and define a function $s: K \rightarrow Y$ by $s(x)=\sum_{i=1}^{n} \lambda_{i}(x) y_{i}$ for all $x \in K$. If $\lambda_{i}(x) \neq 0$ then $x \in \tilde{A}^{-1}\left(y_{i}\right)$ and so $y_{i} \in \widetilde{A}(x) \subseteq A(x)$; by convexity, $s(x) \in A(x)$. The proof of $\Phi \subseteq \mathrm{S}$ is similar.

TheOREM 2.3. (Michael $[25,26])$.

(i) If $V$ is a convex symmetric neighbourhood of 0 and $A \in \mathrm{L}$, then $A+V \in$ $\mathbf{M} \cap \mathbf{S}$;

(ii) if $Y$ is a closed subset of a Fréchet space, then $\mathrm{L}(X, Y) \subseteq \mathrm{S}(X, Y)$;

(iii) if $Y$ is a Banach space, $\mathbf{L}_{w}(X, Y) \subseteq \mathbf{S}(X, Y)$.

\section{EXTENSION SPACES}

We give in this section some definitions and basic properties of extension spaces. For a more comprehensive exposition, the reader is referred to Granas [16] and Hanner [19]. In what follows, $Q$ denotes a class of topological spaces.

DEFINITION 3.1: (i) A space $Y$ is an extension (respectively neighbourhood extension) space for $Q$ if for any pair $(X, K)$ in $Q$ with $K \subseteq X$ closed and any continuous function $f_{0}: K \rightarrow Y$ there is a continuous extension $f: X \rightarrow Y$ (respectively neighbourhood extension $f: U \rightarrow Y$ ) of $f_{0}$ over $X$ (respectively over a neighbourhood $U$ of $K$ in $X$ ). The corresponding classes of extension spaces will be denoted by $\operatorname{ES}(Q)$ and $\operatorname{NES}(Q)$, respectively.

(ii) A space $Y$ is an approximate neighbourhood extension space for $Q$ if for a given covering $\alpha \in \operatorname{Cov}(Y)$ and for any pair $(X, K)$ in $Q$ with $K \subseteq X$ closed and any continuous function $f_{0}: K \rightarrow Y$ there is a neighbourhood $U_{\alpha}$ of $K$ in $X$ and a continuous function $f_{\alpha}: U_{\alpha} \rightarrow Y$ such that $f_{\alpha / K}$ and $f_{0}$ are $\alpha$-close. The class of approximate neighbourhood extension spaces for $Q$ will be denoted by $\operatorname{ANES}(Q)$.

Clearly $\operatorname{ES}(Q) \subseteq \operatorname{NES}(Q) \subseteq \operatorname{ANES}(Q)$ and if $Q \subseteq Q^{\prime}$ then $\operatorname{NES}\left(Q^{\prime}\right) \subseteq$ $\operatorname{NES}(Q)$ and $\operatorname{ANES}\left(Q^{\prime}\right) \subseteq \operatorname{ANES}(Q)$. Some basic properties of extension spaces are listed below:

\section{Proposition 3.2.}

(i) A retract of a member of $\mathbf{E S}(Q)$ belongs to $\operatorname{ES}(Q)$; the same is true of 


\section{$\operatorname{ANES}(Q)$ if $Q$ is compact;}

(ii) a neighbourhood retract of an $\operatorname{NES}(Q)$ is an $\operatorname{NES}(Q)$;

(iii) an open subset of a member of $\operatorname{NES}(Q)$ belongs to $\operatorname{NES}(Q)$;

(iv) the product of any subclass (respectively any finite subclass) of $\operatorname{ES}(Q)$ (respectively $\operatorname{NES}(Q)$ ) belongs to $\operatorname{ES}(Q)$ (respectively $\operatorname{NES}(Q)$ );

(v) if $Q$ is the class of paracompact spaces, then any local member of $\operatorname{NES}(Q)$ is an $\operatorname{NES}(Q)$;

(vi) let $Y=\bigcup_{i=1}^{n} Y_{i}$ with $Y_{i}$ open and $Q$ be a class of normal spaces; then if $Y_{i} \in \operatorname{NES}(Q)$ for all $i$, it follows that $Y \in \operatorname{NES}(Q)$.

We give now some examples of extension spaces (for a more detailed list see [15]).

\section{Proposition 3.3 .}

(i) Let $Q$ be a class of normal spaces, and $\mathbf{A R}(Q)$ (respectively $\operatorname{ANR}(Q)$ ) be the class of absolute retracts (respectively absolute neighourhood retracts) for $Q$, then $\operatorname{AR}(Q)=Q \cap \mathbf{E S}(Q)$ (respectively $\operatorname{ANR}(Q)=$ $Q \cap \operatorname{NES}(Q))$;

(ii) the unit interval $[0,1]$ and the real line $R$ belong to ES (normal) (TietzeUrysohn) and therefore every Tychonoff cube is in ES (normal);

(iii) every convex subset of a locally convex t.v.s(or of a vector space with the finite topology) is in ES (metric) (Dugundji [0]);

(iv) any normed space $E$ is an ES (compact); every complete metric linear space admissible in the sense of Klee [22] is in ES (compact) (in particular, $L^{p}$ for $p \leqslant 1$, and the space $\mathcal{M}$ of measurable functions are in ES (compact));

(v) $\mathbf{A N R}=\mathbf{A N R}$ (metric) $\subseteq$ NES (compact) (therefore any $C W$-complex is in NES (compact); and if $E$ is a locally convex t.v.s., $C=\bigcup_{i=1}^{n} C_{i}$ with $C_{i}$ closed in $E$ is metrisable, then $C$, being in ANR is in NES (compact);

(vi) every open subset and every convex subset of a locally convex t.v.sare in ANES (compact).

\section{FIXED POINTS FOR COMPOSITIONS OF MAPS}

We give in this section several fixed point theorems for compositions of maps which will be used in the next section to derive our basic coincidence theorems. Our main goal here is to give an elementary proof of fixed point theorems for a compact $A \in \mathbf{K}_{c}(X)$, where $X$ is some extension space. 
The initial idea is contained in the following result, proved in [4]. Its proof relies on the existence of an approximate selection.

Proposition 4.1. Given $X$ compact is a t.v.s. $E, Y$ a subset of a t.v.s. $F$, $A \in \mathbf{K}(X, Y)$ and $f \in \mathcal{C}(Y, X)$. If $X \in \mathcal{F}_{\mathcal{C}}$ then $\operatorname{Fix}(f A) \neq \emptyset$.

In fact, a simple adaption of the proof leads to

Proposition 4.2. Given $X$ compact in a t.v.s. $E, Y$ a subset of a t.v.s. $F$, $A \in \mathbf{K}(X, Y)$ and $B: Y \rightarrow X$ a usc map with closed values. If for any $f \in \mathcal{C}(X, Y)$ the composition $B f$ has a fixed point, then $B A$ has a fixed point.

Proof: Let $U \in \vartheta_{F}(0), V \in \vartheta_{E}(0)$ be symmetric. Since $A$ is usc, for any $x \in X$, there exists $V_{x} \in \vartheta_{E}(0)$ such that $x^{\prime} \in x+V_{x} \Rightarrow A\left(x^{\prime}\right) \subseteq A(x)+U$. One can assume with no loss of generality that $V_{x} \subseteq V$ for all $x \in X$. By compactness, $X \subseteq \bigcup_{i=1}^{n} x_{i}+1 / 2 V_{x_{i}}$ and if $W=\bigcap_{i=1}^{n} 1 / 2 V_{x_{i}}$ then $X \subseteq \bigcup_{k=1}^{m} u_{k}+W$. Now, for any $k$ and any $i$ such that $u_{k} \in x_{i}+V_{x_{i}}$ choose $y_{i(k)} \in A\left(x_{i}\right)$. Let $x \in X$ be arbitrary and $i \in\{1, \ldots, n\}$ be such that $x \in x_{i}+1 / 2 V_{x_{i}}$. If $\left\{\lambda_{k}\right\}$ is a partition of unity subordinated to $\left\{u_{k}+W\right\}$ and if $k \in N x=\left\{k \mid \lambda_{k}(x) \neq 0\right\}$ then $u_{k} \in x_{i}+V_{x_{i}}$ and $f(x)=y_{i}=\sum_{k \in N_{x}} \lambda_{k}(x) y_{i(k)} \in A\left(x_{i}\right)$. Since $f$ is continuous, the composition $B f$ has a fixed point $x_{0} \in B f\left(x_{0}\right)$; and since $f\left(x_{0}\right) \in A\left(\widehat{x}_{0}\right)$ for some $\widehat{x}_{0} \in x_{0}+1 / 2 V$, $\widehat{x}_{0} \in B A\left(\widehat{x}_{0}\right)+V$. Since $B A$ is usc with closed values and $V$ is arbitrary, Lemma 1.2 ends the proof.

Using this result, we give a different proof of a result of Lassonde [24].

LEMMA 4.3. Given a non-empty compact $X$ of a t.v.s. $E$, an arbitrary subset $Y$ of a t.v.s. $F$ and a closed subset $\Gamma$ of $X \times Y$, the following statements are equivalent:

(i) for each $f \in \mathcal{C}(X, Y), \Gamma_{f} \cap \Gamma \neq \emptyset$;

(ii) for each $A \in \mathbf{K}_{c}(X, Y), \Gamma_{A} \cap \Gamma \neq \emptyset$.

Proof: (ii) $\Rightarrow$ (i) is trivial. We prove (i) $\Rightarrow$ (ii) by induction. First, notice that, since $X$ is compact, $\Gamma$ can be viewed as the graph of an usc map $B: Y \rightarrow X$ that is $\Gamma_{B}=\Gamma$. Let $A \in \mathbf{K}(X, Y)$; by (4.2), the composition $B A: X \rightarrow X$ has a fixed point. Assume now that (ii) holds for every composition of $n-1 \mathrm{~K}$-maps and let us prove that it is true for the composition

$$
A=A_{n} A_{n-1} \ldots A_{1}: X \stackrel{A_{1}}{\longrightarrow} X_{1} \stackrel{A_{2}}{\longrightarrow} X_{2} \ldots X_{n-1} \stackrel{A_{n}}{\longrightarrow} X_{n}=Y \text { of } n \mathrm{~K} \text {-maps. }
$$

Let $f \in \mathcal{C}\left(X, X_{1}\right)$ be arbitrary. $\widehat{A}_{2}=A_{2} f \in \mathbf{K}\left(X, X_{1}\right)$ and by hypothesis, if $\widehat{A}=$ $A_{n} A_{n-1} \ldots \widehat{A}_{2}$ then $\Gamma_{\widehat{A}} \cap \Gamma \neq \emptyset$ that is $B \widehat{A}$ has a fixed point. If $R=B A_{n} A_{n-1} \ldots A_{2}$, then, $R f$ has a fixed point, that is, $\Gamma_{f} \cap \Gamma_{R} \neq \emptyset$. Now applying Proposition 4.2 again, we obtain that $R A_{1}$ has a fixed point, so $\Gamma_{A} \cap \Gamma \neq \emptyset$. 
This induction argument is due to Lassonde. However, his proof, valid for $X$ a simplex, is based on an approximation by simplicial maps technique.

If $Y=X$ and $\Gamma=\{(x, x) \mid x \in X\}$ is the diagonal and if $E$ is locally convex, it follows from the Schauder-Tychonoff fixed point theorem that:

Theorem 4.4. Let $X$ be a convex compact subset of a locally convex $t$.v.s. $E$ and $A \in \mathbf{K}_{c}(X)$ (the intermediate spaces being arbitrary t.v.s.), then $\operatorname{Fix}(A) \neq \emptyset$.

REMARKS. (1) If all the intermediate spaces are locally convex (Pasicki [27]), the conclusion follows at once from the regularity of the class $\mathbf{K}$ (Lemma 2.1) and from Lemma 1.1 .

(2) Theorem 4.4 remains valid if the compactness is placed on the map. Actually, Lassonde's result extends to a wider class of spaces by using a standard procedure (see for example Górniewicz [13], Granas [16]). Since by Theorem 4.4 every Tychonoff cube $T \in \mathcal{F}_{\mathbf{K}_{c}}$ and since every compact space is homeomorphic to a closed subset of the Tychonoff cube, we are able to prove the following:

TheOREM 4.5. If $X \in \mathbf{E S}$ (compact) and $A \in \mathbf{K}_{c}(X)$ is compact, then $\operatorname{Fix}(A) \neq \emptyset$.

Proof: Let $X \in \mathbf{E S}$ (compact) and $A \in \mathbf{K}_{c}(X)$ be a compact map that is $A(X) \subseteq$ $K$ which is compact in $X$. Let $\widehat{K}$ be a closed subset of the Tychonoff cube $T$, and $s: K \leftrightarrow \widehat{K}$ a homeomorphism. Then we have the commutative diagram

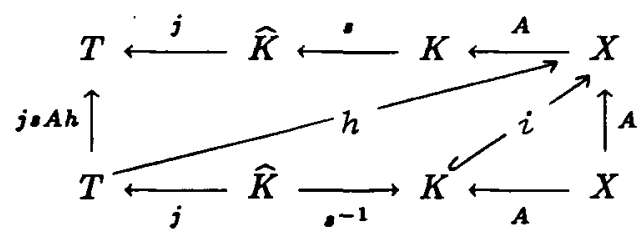

where $i, j$ are the inclusions and $h$ a continuous extension of $i s^{-1}$. A fixed point for $j s A h$ leads to a fixed point for $A$ and the proof is complete.

Before proving the fixed point theorem for $\mathbf{K}_{\mathbf{c}}$-maps defined on a member of NES(compact), let us formulate some preparatory results. We start with:

Lemma 4.6. (see [15]). Let $K$ be a compact subset of a t.v.s. $E$. Then the linear envelope Span $(K)$ of $K$ is Lindelöf (and hence paracompact).

LEMMA 4.7. Let $T$ be a Tychonoff cube contained in a t.v.s. $E$. Then $T$ is a retract of $\operatorname{Span}(T)$.

Proof: Since $T \in \mathbf{E S}$ (normal) (Proposition 3.3 (ii)) and $\operatorname{Span}(T)$, being para- 
compact, is normal, there exists a function $r$ such that the following diagram

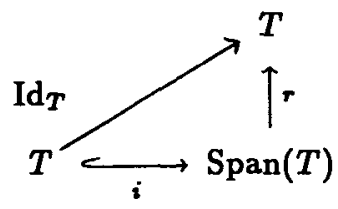

commutes.

Given a finite subset $N=\left\{c_{1}, c_{2}, \ldots, c_{n}\right\}$ of a locally convex t.v.s. $E$, the Schauder projection associated with an open set $U \in \vartheta_{E}(0)$ is the function $\pi_{U}: \bigcup_{i=1}^{n}\left(c_{i}+U\right) \rightarrow E$ given by

$$
\pi_{U}(x)=\frac{\sum_{i=1}^{n} \mu_{i}(x) c_{i}}{\sum_{i=1}^{n} \mu_{i}(x)} \text { for all } x
$$

where $\mu_{i}(x)=\max \left\{0,1-p_{U}\left(x-c_{i}\right)\right\}, p_{U}$ being a semi-norm associated with $U$.

It is easily seen that $\pi_{U}(x)-x \in U$ and that $\pi_{U}(x) \in C-\operatorname{conv}\{N\}$ for all $x$. The next results are immediate:

LEммa 4.8. Let $V$ be an open subset of a locally convex t.v.s. $E$ and $K$ be a compact subset of $V$. Then, for each covering $\alpha \in \operatorname{Cov}_{V}(K)$, there exists a function $\pi_{\alpha}: K \rightarrow V$ such that

(i) $\pi_{\alpha}$ and $i: K \hookrightarrow V$ are $\alpha$-close;

(ii) $\pi_{\alpha}(K)$ is contained in a finite polyhedron $C \subset V$.

Lemma 4.9. Let $V$ be an open subset of a locally convex t.v.s. $E$ and $A \in$ $\mathrm{K}_{c}(V)$ compact; then $A$ has a fixed point.

Proof: Since $A(V) \subseteq K$ is compact, then for each $\alpha \in \operatorname{Cov}_{V}(K)$, there exist a function $\pi_{\alpha}: K \rightarrow V$ and a finite polyhedron $C \subseteq V$ such that $A_{\alpha}(V)=\pi_{\alpha} A(V) \subseteq C$. Hence, by Theorem 4.5, the restriction $A_{\left.\alpha\right|_{C}}: C \stackrel{A}{\longrightarrow} K \stackrel{\pi_{\alpha}}{\longrightarrow} C$ has a fixed point which in turn is a $\alpha$-fixed point for $A$; the conclusion follows from Lemma 1.2.

We are now ready to prove:

Thzorem 4.10. If $X \in \mathrm{NES}$ (compact) and $A \in \mathbf{K}_{\mathrm{c}}(X)$ is compact then $A$ has a fixed point.

Proof: Let $A(X) \subseteq K, \widehat{K}$ be a closed subset of the Tychonoff cube $T$ and $s: K \leftrightarrow \widehat{K}$ be a homeomorphism. Now $X \in$ NES (compact). Let $U$ be an open neighbourhood of $\widehat{K}$ in $T$ and $h: U \rightarrow X$ be a continuous extension of $i s^{-1}: \widehat{K} \rightarrow X$ 
on $U$; then if $\widehat{K} \stackrel{j}{\hookrightarrow} U$ is the natural embedding, $h j=i s^{-1}$. Now consider $\operatorname{Span}(T)$ in a locally convex t.v.scontaining $T$, then Lemma 4.7 ensures the existence of a retraction $r: \operatorname{Span}(T) \rightarrow T$. We can now build the following commutative diagram

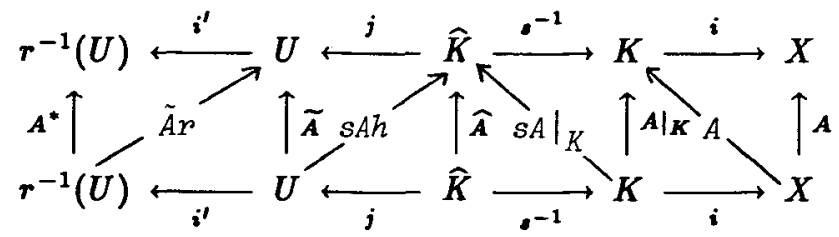

where $\widehat{A}, \widetilde{A}$ are the appropriate maps, $A^{*}=i^{\prime} \tilde{A} r=i^{\prime} j s A h r \in \mathbf{K}_{c}\left(r^{-1}(U)\right)$ is compact and has a fixed point by Theorem 4.10. Lemma 1.3 ends the proof.

We now extend this theorem to the larger class of ANES(compact).

THEOREM 4.11. If $X \in \mathbf{A N E S}$ (compact) and $A \in \mathbf{K}_{c}(X)$ is compact then $A$ has a fixed point.

Proof: Let $A(X) \subseteq K, \widehat{K}$ a closed subset of the Tychonoff cube $T$ and $s: K \leftrightarrow$ $\widehat{K}$ a homeomorphism. We have the following commutative diagram with $A=i A^{\prime}$ and $\widehat{A}=\left.s A\right|_{K} s^{-1}$

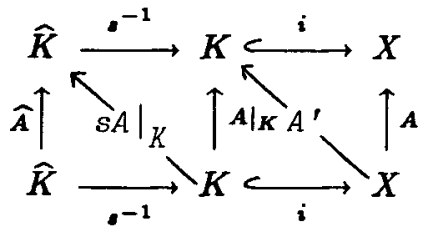

Let $\alpha \in \operatorname{Cov}(X)$ and consider is ${ }^{-1}: \widehat{K} \rightarrow X$; since $X \in$ ANES (compact), there exist an open neighbourhood $U_{\alpha}$ of $\widehat{K}$ in $T$ and $f_{\alpha}: U_{\alpha} \rightarrow X$ a continuous function such that $f_{\alpha}$ and $i s^{-1}$ are $\alpha$-close on $\widehat{K}$; let $\widehat{K} \stackrel{j}{\leftrightarrow} U_{\alpha}$ be the natural imbedding and consider the following commutative diagram

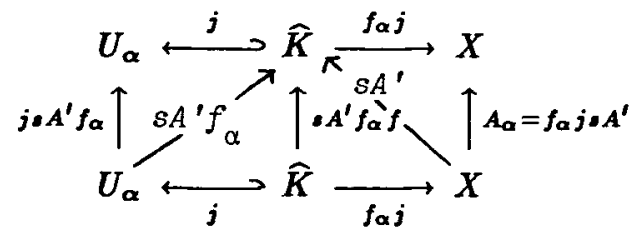

Since $U_{\alpha} \in$ NES (compact), then by Theorem $4.10 \mathrm{Fix}\left(j s A^{\prime} f_{\alpha}\right) \neq \emptyset$ and therefore $\operatorname{Fix}\left(A_{\alpha}\right) \neq \emptyset$. But $f_{\alpha} j$ and $i s^{-1}$ are $\alpha$-close, that is, $A_{\alpha}$ and $A$ are $\alpha$-close and therefore $A$ has an $\alpha$-fixed point. Since $\alpha$ is arbitrary, Lemma 1.2 ends the proof.

This theorem contains the results of Lassonde [23], the Fan-GlicksbergHimmelberg fixed point theorem and holds for some not necessarily locally convex spaces. It was recently formulated using homological methods for an abstract class of maps determined by morphisms and containing $\mathbf{V}_{c}$ by Gòrniewicz and Granas. 


\section{Coincidence Results}

Our first coincidence result follows from the analogue of Theorem 4.10 with $\mathrm{K}_{c}$ replaced by $V_{c}$ (Fournier and Górniewicz [10]) and contains Theorem 0 as a particular case. It was formulated in [6].

THEOREM 5.1. (Coincidence $\left(\mathrm{V}_{c}, \mathrm{M}_{\mathrm{c}}^{-}\right)$). Given a convex subset $X$ of a t.v.s. $E$, a subset $Y$ of a t.v.s. $F$ and two maps $A \in \mathrm{V}_{c}(X, Y)$ and $B \in \mathrm{M}_{c}^{-}(X, Y)$; if $Y$ is compact or $A$ is compact, then $\Gamma_{A} \cap \Gamma_{B} \neq \emptyset$.

Proof: Let $K$ be a compact subset of $Y$ satisfying $A(X) \subseteq K$; by regularity, the restriction $\left.B^{-1}\right|_{K} \in \mathbf{M}_{c}(K, X)=\mathbf{M}(K, X)$. Therefore there exists a finite subset $\left\{x_{1}, x_{2}, \ldots, x_{n}\right\} \subseteq X$ and a function $f \in \mathcal{C}(K, X)$ such that $f(y) \in B^{-1}(y)$ for all $y \in K$ and $f(K) \subseteq C=\operatorname{conv}\left(x_{1}, x_{2}, \ldots, x_{n}\right)$. Since $C$ is compact convex in a finite dimensional subspace of $E$ then $C \in \mathbf{N E S}$ (compact) and is acyclic; hence the composition $\left.f A\right|_{C} \in \mathrm{V}_{c}(C)$ and has a fixed point, that is, there exists $\left(x_{0}, y_{0}\right) \in C \times K$ with $x_{0}=f\left(y_{0}\right) \in B^{-1}\left(y_{0}\right)$ and $y_{0} \in A\left(x_{0}\right)$. This concludes the proof.

If $\mathbf{V}_{c}$ is replaced by $\mathbf{K}_{c}$ (convex case), the result is a direct consequence of Theorem 4.10 .

THEOREM 5.2. Given a convex subset $X$ of a t.v.s. $E$, a subset $Y$ of a t.v.s. $F$ and two maps $A \in \mathbf{K}_{c}(X, Y)$ and $B \in \mathbf{M}_{c}^{-}(X, Y)$; if $A$ is compact, then $\Gamma_{A} \cap \Gamma_{B} \neq \emptyset$.

In the case where $X=Y$ is convex compact and $A$ is the identity, we obtain:

Corollary 5.3. If $X$ is convex compact then $X \in \mathcal{F}_{M}$.

Theorem 5.4. Let $X$ be a convex subset of a t.v.s. $E, Y$ a subset of a t.v.s. $F$, $A \in \mathbf{M}(X, Y)$ compact and $B \in \mathbf{M}^{-}(X, Y)$. Then $\Gamma_{A} \cap \Gamma_{B} \neq 0$.

Proof: Let $K$ be compact such that $A(X) \subseteq K \subseteq Y$. The restriction $\left.B^{-1}\right|_{K} \in$ $\mathrm{M}(K, X)$. Thus, there exist a polytope $C \subseteq X$ and a continuous selection $s \in \mathcal{C}(K, C)$ of $\left.B^{-1}\right|_{K}$. Since $\left.A\right|_{C} \in \mathbf{M}(C, K)$, it admits a selection $f \in \mathcal{C}(C, K)$. By the Brouwer fixed point theorem, the composition $s f: C \rightarrow C$ has a fixed point $x_{0}=s f\left(x_{0}\right)$ and therefore $f\left(x_{0}\right) \in A\left(x_{0}\right) \cap B\left(x_{0}\right)$.

REMARKs. In the case where $F$ is a locally convex t.v.s., $\mathbf{K}_{c}=\mathbf{K}$ in Theorem 5.2, the conclusion follows directly from the regularity of the class $K$ and Lemma 1.1 and we obtain an extension of a result of Browder [8]. In the case where $\mathbf{M}=\mathbf{F}$ or $\Phi$, we obtain results of Deguire and Granas and the author [6], and of Tarafdar [29, 30].

Finally, the following is a direct consequense of Theorem 4.10:

ThEOREM 5.6. Let $X$ be an ANR(compact) and $Y$ be an arbitrary subset of a t.v.s. $F, A \in \mathbf{S}(X, Y)$ and $B \in \mathbf{K}_{c}^{-}(X, Y)$. Then $\Gamma_{A} \cap \Gamma_{B} \neq \emptyset$. 
REMARK. If $X=Y$ is a convex compact subset of a Banach space, $B=\operatorname{Id} X$, and $\mathbf{S}=\mathbf{L}_{w}$, we obtain, as a particular case, a result of Ricceri [28]. Moreover, since $\operatorname{dim}_{X \times Y}\left(Z \times Z^{\prime}\right) \leqslant \operatorname{dim}_{X}(Z)+\operatorname{dim}_{Y}\left(Z^{\prime}\right)$ if one of the sets $Z, Z^{\prime}$ is non-empty, and since the class $S$ satisfies Lemma 1.1, $\Gamma_{A} \cap \Gamma_{B} \neq \emptyset$ for any $A \in \mathbf{L}_{w}(X, Y)$ and $B \in \mathbf{L}_{\mathbf{w}}^{-}(X, Y)$ where $X, Y$ are convex compact subsets of Banach spaces [28].

\section{Special cases}

In some of the theorems of Section 5 , the compactness conditions can be weakened by a coercivity condition on the map.

We say that a map $A: X \rightarrow Y$ satisfies the condition $(\mathcal{K})$ if and only if there exist a compact $K \subseteq X$ and a compact convex $C \subseteq Y$ such that $A(x) \cap C \neq \emptyset$ for any $x \in X \backslash K$. This coercivity condition was first introduced in this form by Deguire, Granas and the author [5] and generalises a condition of Allen [1]. Related or equivalent conditions were considered by various authors to relax the compactness conditions on the domains. The reader is referred to $[3,12,23]$ and $[29,30]$.

In the case where $\mathbf{M}=\boldsymbol{\Phi}$, we formulate the following generalisations:

Theorem 6.1. Let $X$ be a convex subset of a t.v.s. $E, A \in \mathrm{K}(X, Y)$ and $B \in \mathbf{\Phi}^{-}(X, Y)$. If $B$ has an admissible selection $\widetilde{B}$ such that $\widetilde{B}^{-1}$ satisfies $(\mathcal{K})$ then $\Gamma_{A} \cap \Gamma_{B} \neq \emptyset$.

Proof: Let $K \subseteq Y$ be compact and $C \subseteq X$ be convex compact such that $\widetilde{B}^{-1}(y) \cap C \neq \emptyset$ for any $y \in Y \backslash K$. Since $B^{-1} \in \Phi(X, Y) \subseteq \mathbf{M}(X, Y)$, there exist a polytope $C^{\prime} \subseteq X$ and a selection $s \in \mathcal{C}\left(K, C^{\prime}\right)$ of $\left.B^{-1}\right|_{K}$. Consider now the compact convex $\widehat{C}=\operatorname{conv}\left(C, C^{\prime}\right)$ and apply Theorem 5.2 to the maps $\left.A\right|_{\widehat{C}} \in \mathbf{K}(\widehat{C}, Y)$ and $\widehat{B}^{-1}: Y \rightarrow \widehat{C} \in \Phi(Y, \widehat{C})$ defined by $\widehat{B}^{-1}(y)=B^{-1}(y) \cap \widehat{C}$ to get a coincidence which is in turn a coincidence between $A$ and $B$.

As an immediate consequence, one gets the following fixed point theorem:

Corollary 6.2. Let $X$ be convex in a t.v.sand $A \in \Phi(X)$. If $A$ has an admissible selection satisfying $(\mathcal{K})$ then $\operatorname{Fix}(A) \neq \emptyset$.

Corollary 6.3. Let $Y$ be convex in a t.v.s., $A \in \Phi(X, Y)$ and $B \in \Phi^{-}(X, Y)$. If $A$ has an admissible selection satisfying $(\mathcal{K})$ then $\Gamma_{A} \cap \Gamma_{B} \neq \emptyset$.

It is easy to see that Corollary 6.2 is equivalent to the generalised KKM maps principle formulated by Fan in [12] which was expressed by the same author as a matching theorem for open coverings of convex sets. We use this matching theorem to prove a fixed point theorem for the class $\$$ without convexity on the domain [4].

THEOREM 6.4. If $Z$ is a compact and star-shaped subset of a t.v.s. $E$, then $Z \in \mathcal{F}_{\mathbf{F}}$ (and therefore $Z \in \mathcal{F}_{\mathbf{\Psi}}$ ). 
The following problem is open: in the case where $\mathbf{M}=\mathbf{F}$, can we extend Corollary 5.3 to

Given a convex subset $X$ of a t.v.s. $E$ and $A \in \mathbf{F}(X)$ compact then $\operatorname{Fix}(A) \neq \emptyset$ ?

The following partial answer is due to Deguire and Granas and the author [5]. Let $K$ be a compact subset of $X$ containing $A(X)$. Since $\mathbf{F} \subseteq \mathbf{S}$, and since the linear envelope $L$ of $K$ is paracompact then (if $X$ is closed) $L \cap X$ being paracompact, there exists a continuous selection $s$ of $\left.A\right|_{L \cap X}$ and a fixed point for $s$ follows from the Schauder-Tychonoff fixed point theorem, provided $E$ is locally convex.

Without local convexity, it is an easy matter to prove that all the iterates $A^{k}$, $k \geqslant 2$, have a fixed point.

\section{REFERENCES}

[1] G. Allen, 'Variational inequalities, complementarity problems and duality theorems', $J$. Math. Anal. Appl. 58 (1977), 1-10.

[2] J.P. Aubin and A. Cellina, Differential inclusion (Springer-Verlag, Berlin, Heidelberg, New York, 1984).

[3] H. Ben-El-Mechaiekh, Quelques principles topologiques en analyse convexe (Thèse de $\mathrm{PhD}$, Univ. de Montréal, 1988).

[4] H. Ben-El-Mechaiekh, 'Coincidence theorems for set-valued maps', (preprint).

[5] H. Ben-El-Mechaiekh, P. Deguire and A. Granas, 'Une alternative non-linéaire en analyse convex et application', C. R. Accad. Sci Paris Sér 1 Math. 295 (1982), 257-259.

[6] H. Ben-El-Mechaiekh, P. Deguire and A. Granas, 'Points fixes et coincidences pour les fonctions multivoques III (Applications de type $M$ et $M^{*}$ )', C.R. Acad. Sci. Paris, Série I (1987), 305-381.

[7] C. Berge, Espaces topologiques, fonctions multivoques (Dunod, Paris, 1959).

[8] F.E. Browder, 'The fixed point theory of multi-valued mappings in topological vector spaces', Math. Ann. 177 (1968), 283-301.

[9] J. Dugundji, 'An extension of Tietze's theorem', Pacific J. Math. (1951), 353-367.

[10] G. Fournier and L. Górniewicz, 'The Lefschetz fixed point theorem for multivalued maps of non-metrizable spaces', Fund. Math. 92 (1976), 213-222.

[11] K. Fan, 'Fixed point and minimax theorems in locally convex topological linear spaces', Proc. Nat. Acad. Sci. U.S.A. 38 (1952), 121-126.

[12] K. Fan, 'Some properties of convex sets related to fixed point theorems', Math. Ann. 266 (1984), 519-537.

[13] L. Górniewicz, 'Homological methods in fixed-point theory of multi-valued maps', Dissertationes Math.,(Roprawry Mat) CXXXIX (1976).

[14] L. Górniewicz and A. Granas, 'Some general theorems in coincidence theory I', J. Math. Putes Appl. 60 (1981), 361-373.

[15] L. Górniewicz and A. Granas, 'The Lefschetz fixed point theorem for multivalued mappings', paper given at Colloque International Theorie du Point fixe, Marseille. 
[16] A. Granas, Points fixes pour les applications compactes: espaces de Lefschetz et la théorie de l'indice (Presses Univ. de Montréal, 1980).

[17] A. Granas and F.C. Liu, 'Coincidences for set-valued maps and minimax inequalities', $J$. Math. Pures Appl. 65 (1986), 119-148.

[18] C.W. Ha, 'Minimax and fixed point theorems', Math. Ann. 248 (1980), 73-77.

[19] O. Hanner, 'Retraction and extension of mappings of metric and non-metric spaces', Ark.Mat. 2 (1952), 315-360.

[20] W. Hurewicz and H. Wallman, Dimension Theory (Princeton, 1941).

[21] S. Kakutani, 'A generalization of Brouwer's fixed point theorem', Duke Math. J. 8 (1941), 457-459.

[22] V. Klee, 'Leray-Schauder theory without local convexity', Math. Ann. 141 (1960), 286-296.

[23] M. Lassonde, 'On the use of KKM multifunctions in fixed point theory and related topics', J. Math. Anal. Appl 87 (1983), 151-201.

[24] M. Lassonde, 'Fixed points for Kakutani factorizable multifunctions', (Preprint, 1989).

[25] E. Michael, 'Continuous selections', Ann. of Math. 63 (1956), 361-382.

[26] E. Michael, 'Continuous selections and countable sets', Fund. Math. 111 (1981), 1-10.

[27] L. Pasicki, 'Composition of compact mappings has a fixed point', (Preprint, 1980).

[28] B. Ricceri, 'Fixed points of lower semicontinuous multifunctions and applications: alternative and minimax theorems', Rend. Accad. Naz. XL, Mem. Mat. 16 (1985), 331-338.

[29] E. Tarafdar, 'A fixed point theorem equivalent to Fan-Knaster-KuratowskiMazurkiewicz's theorem', J. Math. Anal. Appl. 128 (1987), 475-479.

[30] E. Tarafdar, 'On two theorems concerning sets with convex sections', Bull. Austral. Math. Soc. 38 (1988), 397-400.

[31] L. Vietoris, 'Ueber den höheren zusammenhang kompakier raume und eine kiasse von zusammenhangstrenen abbidungen', Math. Ann. 97 (1927), 454-472.

\author{
Department of Mathematics \\ Brock University \\ St Catharines, Ontario L2S 3A1 \\ Canada
}

